Published in final edited form as:

Injury. 2017 January ; 48(1): 177-183. doi:10.1016/j.injury.2016.11.022.

\title{
Diagnostic accuracy of the Kampala Trauma Score using estimated Abbreviated Injury Scale scores and physician opinion
}

\author{
Andrew Gardner, BS, \\ Medical School, University of Michigan, Ann Arbor, USA \\ Paa Kobina Forson, MB, ChB, \\ Department of Emergency Medicine, Kwame Nkrumah University of Science and Technology, \\ Kumasi, Ghana \\ George Oduro, MB, ChB, \\ Department of Emergency Medicine, Kwame Nkrumah University of Science and Technology, \\ Kumasi, Ghana
}

\section{Barclay Stewart, MD, MscPH,}

Department of Surgery, University of Washington, Seattle, USA; Department of Surgery, Kwame Nkrumah University of Science and Technology, Kumasi, Ghana

\author{
Nkechi Dike, MB, \\ Department of Emergency Medicine, Kwame Nkrumah University of Science and Technology, \\ Kumasi, Ghana
}

Paul Glover, MB, and

Department of Emergency Medicine, Kwame Nkrumah University of Science and Technology, Kumasi, Ghana

Ronald F. Maio, DO, MS

Department of Emergency Medicine, University of Michigan, Ann Arbor, USA

\section{Abstract}

Background-The Kampala Trauma Score (KTS) has been proposed as a triage tool for use in low- and middle-income countries (LMICs). This study aimed to examine the diagnostic accuracy of KTS in predicting emergency department outcomes using timely injury estimation with Abbreviated Injury Scale (AIS) score and physician opinion to calculate KTS scores.

Methods-This was a diagnostic accuracy study of KTS among injured patients presenting to Komfo Anokye Teaching Hospital A\&E, Ghana. South African Triage Scale (SATS); KTS

Corresponding author: Andrew Gardner, University of Michigan Medical School, 2800 Plymouth Road, Suite G080, NCRC Building 10, Ann Arbor, MI 48109-2800, Phone: 208-914-0331, Fax: 734-936-2706, aellisgardner@ gmail.com.

Conflict of interest: No conflicts of interest are declared.

Publisher's Disclaimer: This is a PDF file of an unedited manuscript that has been accepted for publication. As a service to our customers we are providing this early version of the manuscript. The manuscript will undergo copyediting, typesetting, and review of the resulting proof before it is published in its final citable form. Please note that during the production process errors may be discovered which could affect the content, and all legal disclaimers that apply to the journal pertain. 
component variables, including AIS scores and physician opinion for serious injury quantification; and ED disposition were collected. Agreement between estimated AIS score and physician opinion were analyzed with normal, linear weighted, and maximum kappa. Receiver operating characteristic (ROC) analysis of KTS-AIS and KTS-physician opinion was performed to evaluate each measure's ability to predict A\&E mortality and need for hospital admission to the ward or theatre.

Results-A total of 1,053 patients were sampled. There was moderate agreement between AIS criteria and physician opinion by normal $(\kappa=0.41)$, weighted $\left(\kappa_{\text {lin }}=0.47\right)$, and maximum $\left(\kappa_{\max }=0.53\right)$ kappa. A\&E mortality ROC area for KTS-AIS was 0.93 , KTS-physician opinion 0.89, and SATS 0.88 with overlapping 95\% confidence intervals (95\%CI). Hospital admission ROC area for KTS-AIS was 0.73 , KTS-physician opinion 0.79 , and SATS 0.71 with statistical similarity. When evaluating only patients with serious injuries, KTS-AIS (ROC 0.88) and KTSphysician opinion (ROC 0.88) performed similarly to SATS (ROC 0.78) in predicting A\&E mortality. The ROC area for KTS-AIS (ROC $0.71 ; 95 \%$ CI 0.66-0.75) and KTS-physician opinion (ROC 0.74; 95\%CI 0.69-0.79) was significantly greater than SATS (ROC 0.57; 0.53-0.60) with regard to need for admission.

Conclusions-KTS predicted mortality and need for admission from the ED well when early estimation of the number of serious injuries was used, regardless of method (i.e. AIS criteria or physician opinion). This study provides evidence for KTS to be used as a practical and valid triage tool to predict patient prognosis, ED outcomes and inform referral decision-making from first- or second-level hospitals in LMICs.

\section{Keywords}

triage; trauma; trauma severity indices; Kampala Trauma Score; global surgery

\section{Introduction}

Trauma accounts for $11 \%$ of all disability-adjusted life years incurred annually, and disproportionately affects low- and middle-income countries (LMICs) ${ }^{1,2}$. Significant reductions in avertable death and disability can be won with improvements to the quality and processes of care for the injured ${ }^{3}$. Process improvements may be particularly important during emergency care, which is underdeveloped in many $\mathrm{LMICs}^{4,5}$.

Strategies to rapidly and effectively triage the injured are vital to care for patients with appropriate resources in a timely manner ${ }^{6}$. The Kampala Trauma Score (KTS) has been proposed as a potentially useful triage tool for $\mathrm{LMICs}^{7-9}$. KTS may be more practical than other triage tools, such as the South African Triage Scale (SATS), since it requires fewer variables for calculation, and a single algorithm can be applied to all age groups ${ }^{10,11}$. KTS was originally developed as an injury severity score in settings without advanced diagnostics; it has comparable sensitivity and specificity with the Revised Trauma Score (RTS), Injury Severity Score (ISS), and Trauma and ISS (TRISS) for predicting outcomes retrospectively ${ }^{10,12}$. KTS is calculated using the patient's age, systolic blood pressure, respiratory rate, AVPU neurologic status (i.e., A - Alert, V - responds to Voice, P - responds to Pain, $\mathrm{U}$ - Unresponsive), and number of serious injuries ${ }^{10}$. 
Studies that attempted to validate KTS prospectively have used a variety of methods for determining number of serious injuries ${ }^{7-9}$. Further, these reports have not described data collection under the pressure of time, which is inherent to emergency care ${ }^{7-9}$. For the KTS to be a useful triage tool, emergency care teams would have to estimate the number of serious injuries early in the care process. Abbreviated Injury Scale (AIS) scores, and resultant ISS scores, have been successfully used to estimate injury severity, but have not been validated in settings with limited diagnostics ${ }^{13}$. Until now, prospective KTS scoring has been coupled to AIS criteria for determining number of serious injuries ${ }^{8,9}$. A simpler method to quantify serious injuries would potentially make KTS a useful triage tool.

To address these challenges, we sought to evaluate the use of KTS as a triage tool by collecting all component variables upon completion of the secondary survey in a Ghanaian emergency department. In addition, early estimated AIS scores were compared to physician opinion as a more practical method for estimating the number of serious injuries. Lastly, the diagnostic accuracy of KTS performance was compared to the South African Triage Scale (SATS). By doing so, the utility of KTS as a prospective triage tool in the absence of wellresourced diagnostic capacity could be determined.

\section{Methods}

\section{Setting}

This study was conducted at the Accident and Emergency Unit (A\&E) of Komfo Anokye Teaching Hospital (KATH), a tertiary facility, in Kumasi, Ghana. KATH A\&E admits around 14,000 injured children and adults each year, and these patients arrive from the scene of injury as well as lower-level hospitals ${ }^{5}$. All patients are triaged by nurses using SATS. SATS is a validated, reliable, sensitive, and specific 5-level triage acuity scale that assigns each patient to a color-coded acuity tier: i) red-emergency; ii) orange - very urgent; iii) yellow urgent; iv) green - not urgent; and v) blue - dead on arrival ${ }^{11,14,15}$. SATS triages patients based on age, basic physiologic data, neurologic status, and predefined clinical discriminators (such as uncontrolled bleeding). This triage system was designed for lower resourced settings, and as such, has been utilized in various LMICs. ${ }^{16-18}$ Patients triaged green are cared for in a separate unit given their low acuity. KATH A\&E diagnostic capacity is limited due to high-demand, long periods of breakage of equipment, and prohibitively expensive user-fees for some items and services ${ }^{19}$. However, physician physical examination, hemoglobin determination, X-ray, and focused assessment with sonography for trauma (FAST) are routinely available for most injured patients.

\section{Study design and patient selection}

A nested diagnostic accuracy study of KTS among consecutive patients admitted to the KATH A\&E from November 2014 through April 2015 was performed. The subjects in this study were the same as the parent cohort study, which examined the frequency of alcoholrelated trauma. Thus, the inclusion and exclusion criteria were based on breathalyzer and saliva strip alcohol testing limitations. As such, patients aged 18 years and older with kinetic or thermal injuries defined by ICD-10 were included. Patients admitted eight hours or more after they sustained the injury were excluded given concern for complete alcohol metabolism 
and inappropriate classification of alcohol-injured patients. The current study included patients regardless of the alcohol test result. Lastly, patients triaged green were excluded since they were not managed by the A\&E.

\section{Data collection}

Full-time, trained research assistants collected data using Epi Info 7 (Centers for Disease Control, USA) on computer tablets. Research assistants were fluent in both English and Twi, the local language. Data were uploaded to a central computer weekly and checked at random by one of the study investigators to assess completeness of data collection.

Care providers recorded patient data on triage sheets and physician sheets. These sheets were standardized, and prompted providers to consistently record information in the medical records. The following variables were collected by research assistants from chart review: date and time of admission; age, sex; SATS color; systolic blood pressure; respiratory rate; AVPU neurologic status; cause of injury; intent of injury (i.e. unintentional, assault, selfinflicted, undetermined) type of injury; and disposition of the patient from the A\&E (i.e. discharged, admitted to ward, admitted to theatre, died in the A\&E).

Triage nurses determined systolic blood pressure, respiratory rate, and AVPU status. Cause and intent of injury were defined by an ICD-10 injury matrix ${ }^{20}$. Type(s) of injury was collected for nine body regions using abridged AIS-based drop-down menus in the data capture software. These menus were designed to calculate estimated AIS scores in a triage setting. AIS-based menus were developed by condensing the AIS 2005 Update 2008 Manual into non-serious (i.e. AIS 1-2) and serious (i.e. AIS $\geq 3$ ) injuries (Appendix 1) ${ }^{21}$. This version of the manual developed the ability to condense types of injuries through use of a 'not further specified' category ${ }^{21}$. Menu creation was also guided by the limited availability of diagnostics and the types of injuries that commonly present to KATH.

Research assistants completed data collection at the time of the secondary survey. Efforts were made by the care providers to perform the secondary survey immediately after the primary survey, start of resuscitation and results of initial diagnostic tests (e.g. FAST, radiographs, and hemoglobin). Since timely data collection was vital for prospective assessment of KTS, a study investigator was always available to field questions from research assistants (i.e., 24 hours per day, 7 days per week, including holidays).

\section{Physician opinion}

The research assistants then asked the patient's emergency physician to estimate the number of serious injuries. The research assistants provided a standardized definition of serious injury: an injury that is potentially life threatening or may significantly impact the patient's life. This simple definition was used to obtain a response based on physician judgment alone (i.e. not guided by any standardized physiologic or anatomic criteria). Clinicians estimating serious injuries were blinded to AIS and resultant KTS. 


\section{Data collection for sensitivity analysis of excluded patients}

To assess the potential for selection bias with regard to the current study, we retrospectively collected data on patients who were excluded from the parent study. Specifically, data from all adult injury patients who were excluded by category (i.e. patients presenting greater than eight hours from injury, triaged green, or missed the study eligibility screening process) were collected. However, data collection was limited to review of the triage admission book, which only contained: date and time of admission, sex, age, cause of injury category (i.e. road traffic injury, fall, other), and SATS triage color. Of note, cause of injury among excluded patients was only collected from January 1 to April 11, 2015 as a result of paper triage record limitations.

\section{Data analysis}

Date and time of admission, age, sex, SATS color, and mechanism of injury were described for included and excluded patients to examine potential selection bias. Patients' KTS-AIS, KTS-physician opinion, and RTS were plotted to examine distribution and potential outliers. RTS was included in data analysis since this severity measure has been used for comparison in similar diagnostic accuracy studies of $\mathrm{KTS}^{7,8}$. Additionally, patient disposition was tabulated for patients by KTS-AIS and KTS-physician opinion, and SATS. KTS scores were dichotomized according to serious injury scores traditionally defined as less than or equal to fourteen ${ }^{10}$. Scores were negatively skewed; therefore, medians and results of nonparametric analyses were reported. There were no significant outliers. The agreement between early estimated AIS score and physician opinion with regard to the number of serious injuries was assessed by normal, linear weighted, and maximum kappa ${ }^{22,23}$. Using each of these tests allowed for analysis of agreement between the two measures, as well as the loss of agreement due to asymmetric distribution of disagreement (i.e. determination of serious injury by AIS criteria but not physician opinion and vice versa). The sensitivity and specificity of serious injury by estimated AIS score and physician opinion for predicting mortality and need for hospital admission (i.e. admission to inpatient ward or theatre) were evaluated using nonparametric receiver operating characteristics (ROC) analysis. Two-way scatter plots with linear regression were constructed individually for KTS-AIS and KTSphysician opinion with SATS and RTS in order to assess potential correlation. Individual ROC curves for KTS-AIS, KTS-physician opinion, SATS, and RTS were constructed to compare prediction of mortality and need for hospital admission for each score among all study patients and only those with greater injury acuity (i.e. SATS orange through red) in order to evaluate each measure's ability to discern outcomes among severely injured patients. Data were analyzed using Stata v13 (StataCorps; College Station, TX, USA).

\section{Ethics}

The Kwame Nkrumah University of Science and Technology Committee for Human Research and Publication Ethics and the University of Michigan Medical School Institutional Review Board approved this study. Informed consent was waived due to minimal risks to the patient and often impaired or seriously injured status. 


\section{Results}

\section{Patient characteristics}

A total of 2,426 injured patients were triaged at KATH A\&E during the study period. Of these, 1,219 patients (51\%) were excluded, mainly due to prolonged pre-hospital time (869 patients; $71 \%$ of excluded patients) and patients triaged SATS green with minor injuries (198 patients; $21 \%$ of excluded patients). One hundred and thirty-two patients (5\%) were not screened for study eligibility. Twenty-two patients meeting inclusion criteria did not have complete data for this analysis. Resultantly, data from 1,053 patients (43\%) were analyzed. Excluded trauma patients triaged SATS yellow through red were not markedly different from those that were included with regards to time of admission, age, sex, or SATS color (Table 1). There were less RTIs among excluded patients triaged SATS yellow through red.

The median time of admission was 3:45pm (interquartile range (IQR) 11:08am-7:50pm) with median pre-hospital time of 2.0 hours (IQR 1-4 hours). The median age was 33 years (IQR 26-42 years) and most patients were male. The majority of patients were triaged SATS yellow. Road traffic injuries (549 patients; 52\%), falls (172 patients; 16\%), penetrating injuries (160 patients; 15\%), and other blunt injuries (154 patients; 15\%) accounted for $99 \%$ of all injuries. Most injuries were unintentional (879 patients; $84 \%)$.

\section{Injury scores and outcomes}

The median KTS-AIS and KTS-physician opinion scores were similar: median KTS-AIS was 16 (IQR 15-16; range 5-16) and median KTS-physician opinion was 15 (IQR 15-16; range 5-16). The median RTS was 7.84 (IQR 7.84; range 0-7.84). Fifteen patients (1\%) died in the A\&E, 392 (37\%) were admitted to the hospital (i.e. hospital ward or theatre), and 646 (62\%) were discharged home. Table 2 shows the distribution of KTS-AIS scores, KTSphysician opinion scores, and SATS color by patient disposition.

\section{AIS criteria and physician opinion}

There were 307 patients (29\%) with at least one serious injury by AIS criteria compared to 538 patients $(51 \%)$ by physician opinion $(\mathrm{p}<0.001)$. There was moderate agreement using normal kappa $(\kappa=0.41)$ and linear weighted kappa $\left(\kappa_{\text {lin }}=0.47\right)$, respectively. Maximum kappa demonstrated a slight increase in agreement $\left(\kappa_{\max }=0.53\right)$, indicating that there was significant loss of agreement above chance due to asymmetric distribution of disagreement between the two scores. The asymmetry of disagreement was evident when patients with at least one serious injury were evaluated: 17 patients with at least one serious injury by AIS criteria (6\%) were classified as non-serious by physician opinion, whereas 248 patients with at least one serious injury by physician opinion (46\%) were non-serious by AIS criteria $(\mathrm{p}<0.001)$.

AIS criteria and physician opinion performed similarly well predicting A\&E mortality, with ROC areas of 0.92 (95\% CI $0.88-0.97)$ and 0.85 (95\% CI $0.74-0.95)$, respectively. The same was true for prediction of hospital admission from the A\&E; ROC area for AIS criteria was $0.75(95 \% \mathrm{CI} 0.72-0.77)$ and physician opinion was 0.80 (95\% CI $0.77-0.82)$. 
Importantly, the two measures had complementary performance. AIS criteria tended to be more specific while physician opinion tended to have greater sensitivity (Table 3).

\section{Prognostic performance of KTS}

A\&E mortality ROC areas for KTS-AIS, KTS-physician opinion, and SATS were statistically similar, as described in Figure 1. Figure 2 depicts the ROC areas for patients with injuries that required hospital admission. KTS-physician opinion performed better than SATS in predicting need for hospital admission $(\mathrm{p}<0.001)$. These findings are summarized in Table 4. RTS was similar to all other measures when predicting A\&E mortality, but was least able to predict need for admission (Table 4).

\section{Prognostic performance of KTS among high-acuity patients}

Analysis was performed for patients presenting with higher acuity (SATS orange and red) in order to evaluate diagnostic accuracy among those with more severe injuries. For this subset of patients, KTS-AIS and KTS-physician opinion performed similarly to SATS in predicting A\&E mortality (Table 4). Regarding need for hospital admission, the ROC area for KTSAIS and KTS-physician opinion performed statistically greater than SATS.

\section{Discussion}

This study aimed to examine the utility of KTS as a triage tool among patients with moderate-to-severe injuries using early estimation of serious injuries by AIS criteria and physician opinion. We also sought to compare KTS with a validated triage tool and physiologic injury severity measure to further assess its prognostic strength. These results demonstrate that KTS had clinically significant ability to predict need for hospital admission, regardless of whether serious injury was determined by physician opinion or estimated AIS score. KTS was similar to SATS in predicting A\&E mortality and performed equivalently or better than SATS when predicting need for hospital admission in this setting. This performance was also observed among high-acuity patients, and suggests KTS may retain reasonable ability to discern outcomes from the emergency care setting among patients with more severe injuries. Additionally, the KTS demonstrated equivalent to superior diagnostic accuracy when compared to RTS, a severity measure that has previously been used for KTS comparison ${ }^{7,8}$. Finally, the large areas under the mortality and hospital admission ROC curves suggest that KTS may be an effective triage tool when used prospectively. These findings provide support for KTS use after initial physician evaluation to inform prognosis and decisions about potential level of care required or need for referral.

Though KTS has recently been proposed as a potential triage tool, methods for determining the number of serious injuries have not reflected the pressures of limited diagnostics and time in LMIC EDs ${ }^{7-9}$. This study provides initial evidence for the use of KTS as a practical and valid triage tool with early serious injury estimation. Although the estimation of serious injuries in this study was more timely than others to date, KTS in this capacity is not a traditional triage tool because it necessitates physician contact for estimating the number of serious injuries ${ }^{11}$. However, these data suggest KTS may be useful for re-triaging patients, 
or perhaps more importantly, to inform referral decision-making at first- or second-level hospitals after patients have received an initial evaluation.

The equivalent performance of estimated AIS scores with physician opinion in this setting substantially simplifies KTS calculation. By doing so, the feasibility of KTS as a triage tool is significantly improved by use of such consolidated AIS scoring or physician opinion. Although, KTS-AIS and KTS-physician opinion equally predicted A\&E mortality and need for admission, the component AIS criteria tended to have greater specificity while physician opinion tended to have greater sensitivity. This might be explained by the difference in the proportion of patients determined to have at least one serious injury. In the A\&E setting, improved sensitivity is traditionally prioritized to avoid diverting severely injured patients to a lower resourced ward or delaying referral to a higher level of care ${ }^{6}$. Therefore, when utilizing KTS as a triage tool, physician opinion might be preferred over AIS criteria, which has under-predicted mortality in other lower resourced settings ${ }^{24-26}$.

Though the results of this and other studies support the potential of the KTS as a triage tool, additional key steps are needed to further evaluate its practicality as a prognostic tool ${ }^{7-9}$. First, no studies have proposed prospective KTS score cutoffs to stratify injury severity, which is necessary to identify appropriate thresholds for decision-making. Second, KTS could improve the referral process through identification of serious injuries requiring higher level facilities, which is an essential component of the trauma care process ${ }^{27}$. However, the validity of early injury estimation at lower-level hospitals must be assessed since these settings have less resources than tertiary hospitals, which may alter the reliability of such estimations ${ }^{19}$. The validity of our consolidated AIS scores also needs formal validation as this may provide a more replicable framework across varied settings through standardization than physician opinion. Finally, if KTS were to be considered as a traditional triage tool (i.e. used before physician evaluation), the validity of nurse or emergency technician opinion regarding the number of serious injuries would need to be determined.

This study has several strengths and limitations worth mentioning. The study was relatively large, allowing for a robust comparison of injury severity measures. There was not a significant difference between included and excluded patients, providing evidence for only limited potential selection bias despite a large number of excluded patients. Selection bias was further mitigated by minimal loss of patients to follow-up. With regard to limitations, four merit further discussion. First, use of consolidated AIS-based drop-down menus was a novel approach for streamlining AIS estimation of serious injury. While the menus made AIS measurement less granular, we do not feel it substantially impacted the ability to estimate non-serious (i.e. AIS 2 ) from serious (i.e. AIS 23 ) injuries in a resource constrained setting. Additionally, AIS has built-in mechanisms for condensing injuries by the "not further specified (NFS)' option, which was utilized to maintain fidelity. However, utilization of the NFS category tends to be less severe than more specific descriptions. This may have contributed to AIS criteria and physician opinion describing different frequencies of patients with at least one serious injury. Second, despite the relatively large study size, there were a low number of mortalities in the A\&E. This limits the strength in which mortality related results can be interpreted. Third, the median KTS and RTS were relatively high in this study, likely reflecting increased mortality of severely injured patients prior to admission as a result 
of prolonged pre-hospital time common in Ghana and most other LMICs ${ }^{28}$. Validation of this approach in contexts with greater injury severity is required before assessing their generalizability, though sub-set analysis of high-acuity patients is optimistic. Lastly, patients with especially prolonged pre-hospital times (i.e. greater than eight hours) were excluded from this study. This would not be expected to impact the comparative performance of KTS and SATS, since both instruments studied share similar dynamic physiologic measures; anatomic severity is not impacted by time. Despite these limitations, these data allow reasonable conclusions to be made about the prospective use of KTS in this resourceconstrained A\&E with the use of physician opinion to estimate the number of serious injuries for patients with moderate-to-severe injuries.

\section{Conclusions}

KTS performed comparatively and absolutely well to SATS in predicting mortality and need for hospital admission at a tertiary emergency unit in Ghana by using early estimation of serious injury rather than final assessment. Further, using physician opinion at the time of the secondary survey to quantify serious injury is promising, as it performed similar to AIS estimation in this setting. Together, these data provide evidence for the use of KTS as a practical and valid tool to reevaluate patient prognosis during emergency care; thus, KTS may be useful for informing referral practices from first- or second-level hospitals in LMICs. However, further investigation is warranted to evaluate its strength in other settings, such as lower-level hospitals.

\section{Supplementary Material}

Refer to Web version on PubMed Central for supplementary material.

\section{Acknowledgments}

Funding: This study was funded by grant R25-TW009345 from US National Institutes of Health and Fogarty International Center (AG and BTS). The content is solely the responsibility of the authors and does not necessarily represent the official views of the National Institutes of Health.

The authors would like to thank Sonia Cobold, Joycelyn Sarfo-Frimpong, Juliana Kwarteng, Anthony Fosu, Hagar Baidoo, Dorcas Boakye, Abena Boatamaa Boateng, Lydia Offeibea Sakye, Sylvia Adomako, Irene Kutin, Akosua Kwaa Marfo, Belinda Dufie Gyesi, Gifty Ofori, Priscilla Boateng, Zipporah Yong Kukubor Ntekor, Emmanuel Amoateng, Simon Manu, Margaret Agyemang Badu, Irene Nyanor, Vida Odum, Alice Adomako, and Susan Sally Asabea for their support and contributions to the study. This study was funded by grant R25TW009345 from US National Institutes of Health and Fogarty International Center (AG and BTS). The content is solely the responsibility of the authors and does not necessarily represent the official views of the National Institutes of Health.

\section{References}

1. Peden, M.; McGee, K.; Sharma, G. The injury chart book: a graphical overview of the global burden of injuries. Geneva: World Health Organization; 2002. p. 5

2. Mock C. Confronting the global burden of surgical disease. World journal of surgery. 2013; 37(7): 1457-1459. [PubMed: 23674258]

3. Mock, C.; Juillard, C.; Brundage, S.; Goosen, J.; Joshipura, M. Guidelines for trauma quality improvement programs. Geneva, Switzerland: World Health Organization; 2009. 
4. Wesson HK, Bachani AM, Wekesa JM, Mburu J, Hyder AA, Stevens KA. Assessing trauma care at the district and provincial hospital levels: a case study of hospitals in Kenya. Injury. 2013; 44:S75S80. [PubMed: 24377784]

5. Osei-Ampofo M, Oduro G, Oteng R, Zakariah A, Jacquet G, Donkor P. The evolution and current state of emergency care in Ghana. African Journal of Emergency Medicine. 2013; 3(2):52-58.

6. Rotondo, M.; Cribari, C.; Smith, RS. Committee on Trauma. American College of Surgeons; 2014. Resources for optimal care of the injured patient.

7. Haac B, Varela C, Geyer A, Cairns B, Charles A. The utility of the Kampala trauma score as a triage tool in a sub-Saharan African trauma cohort. World J Surg. 2015; 39(2):356-362. [PubMed: 25315093]

8. Weeks SR, Juillard CJ, Monono ME, et al. Is the Kampala trauma score an effective predictor of mortality in low-resource settings? A comparison of multiple trauma severity scores. World J Surg. 2014; 38(8):1905-1911. [PubMed: 24715042]

9. Owor, G.; Kobusingye, OC. Trauma registries as a tool for improved clinical assessment of trauma patients in an urban African hospital. 2007.

10. Kobusingye OC, Lett RR. Hospital-based trauma registries in Uganda. J Trauma. 2000; 48(3):498502. [PubMed: 10744292]

11. Wallis LA, Group CT. The Cape Triage Score: update. Emerg Med J. 2006; 23(9):740.

12. MacLeod JB, Kobusingye O, Frost C, Lett R, Kirya F, Shulman C. A comparison of the Kampala Trauma score (KTS) with the revised Trauma score (RTS), Injury Severity Score (ISS) and the TRISS method in a Ugandan Trauma registry. European Journal of Trauma. 2003; 29(6):392-398.

13. McLellan BA, Koch JP, Wortzman D, Rogers C, Szalai J, Williams D. Early identification of highrisk patients using the "estimated" injury severity score and age. Accident Analysis \& Prevention. 1989; 21(3):283-290. [PubMed: 2736025]

14. Twomey M, Wallis LA, Thompson ML, Myers JE. The South African Triage Scale (adult version) provides reliable acuity ratings. International emergency nursing. 2012; 20(3):142-150. [PubMed: 22726946]

15. Twomey M, Wallis LA, Thompson ML, Myers JE. The South African Triage Scale (adult version) provides valid acuity ratings when used by doctors and enrolled nursing assistants. African Journal of Emergency Medicine. 2012; 2(1):3-12.

16. Twomey M, Mullan PC, Torrey SB, wallis L, Kestler A. The Princess Marina Hospital accident and emergency triage scale provides highly reliable triage acuity ratings. Emerg Med J. 2012; 29(8): 650-653. [PubMed: 21856994]

17. Harrison HL, Raghunath N, Twomey M. Emergency triage, assessment and treatment at a district hospital in Malawi. Emerg Med J. 2012; 29(11):924-925. [PubMed: 22158531]

18. Dalwai MK, Twomey M, Maikere J, et al. Reliability and accuracy of the South African Triage Scale when used by nurses in the emergency department of Timergara Hospital, Pakistan. S Afr Med J. 2014; 104(5):372-375. [PubMed: 25212207]

19. Ankomah, J.; Stewart, BT.; Oppong-Nketia, V., et al. Journal of pediatric surgery. 2015. Strategic assessment of the availability of pediatric trauma care equipment, technology and supplies in Ghana.

20. Annest, J.; Hedegaard, H.; Chen, L-H.; Warner, M.; Smalls, EA. Proposed Framework for Presenting Injury Data using ICD-10-CM External Cause of Injury Codes. Atlanta, GA: National Center for Injury Prevention and Control, National Center for Health Statistics, Centers for Disease Control and Prevention; 2014.

21. Gennarelli, T.; Wodzin, E. Abbreviated Injury Scale 2005. Des Plaines, Illinois: Association for the Advancement of Automotive Medicine; 2008. Update 2008

22. Brenner H, Kliebsch U. Dependence of weighted kappa coefficients on the number of categories. Epidemiology. 1996:199-202. [PubMed: 8834562]

23. Lantz CA, Nebenzahl E. Behavior and interpretation of the $\kappa$ statistic: Resolution of the two paradoxes. Journal of clinical epidemiology. 1996; 49(4):431-434. [PubMed: 8621993]

24. Zafar H, Rehmani R, Raja AJ, Ali A, Ahmed M. Registry based trauma outcome: perspective of a developing country. Emerg Med J. 2002; 19(5):391-394. [PubMed: 12204982] 
25. Hariharan S, Chen D, Parker K, et al. Evaluation of trauma care applying TRISS methodology in a Caribbean developing country. J Emerg Med. 2009; 37(1):85-90. [PubMed: 18584995]

26. Onwudike M, Olaloye OA, Oni OO. Teaching hospital perspective of the quality of trauma care in Lagos, Nigeria. World J Surg. 2001; 25(1):112-115. [PubMed: 11213150]

27. Mock, C.; Lormand, J-D.; Goosen, J.; Joshipura, M.; Peden, M. Guidelines for essential trauma care. Geneva, Switzerland: World Health Organization; 2004.

28. Mock CN, Jurkovich GJ, Arreola-Risa C, Maier RV. Trauma mortality patterns in three nations at different economic levels: implications for global trauma system development. Journal of Trauma and Acute Care Surgery. 1998; 44(5):804-814. 


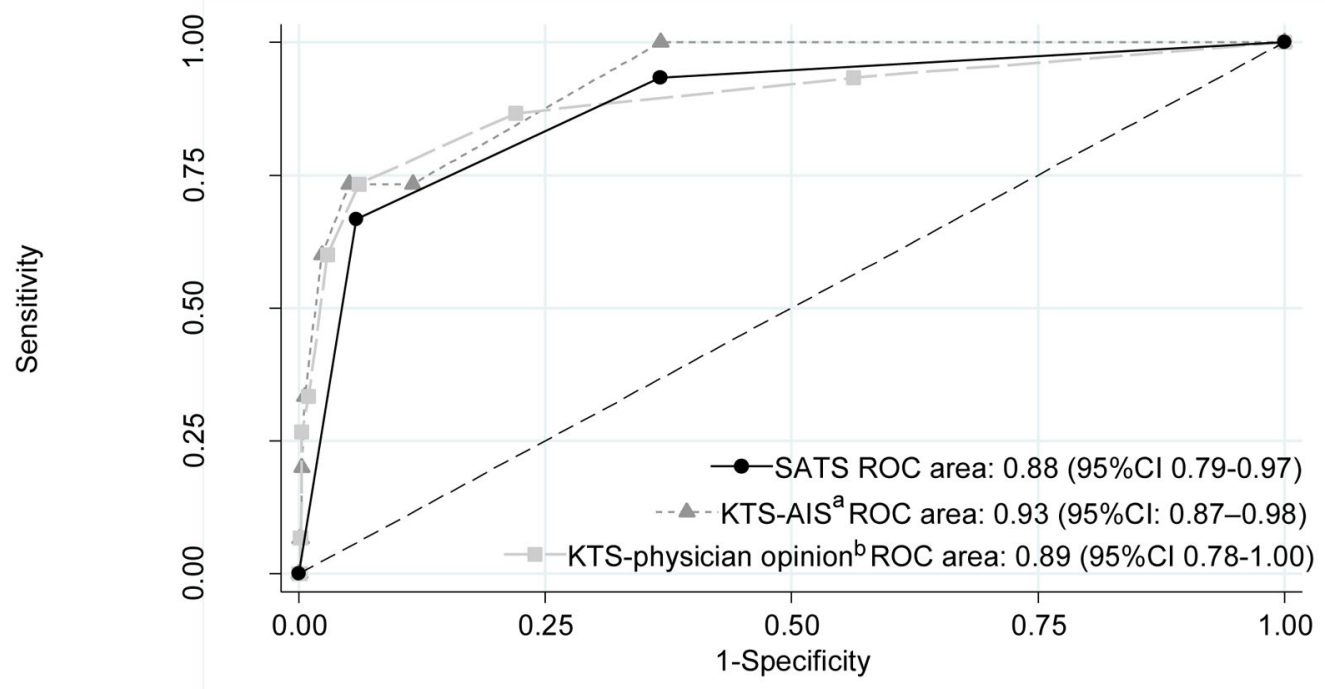

Figure 1. Emergency Department mortality receiver operating characteristics curves for South African Triage Scale (SATS), Kampala Trauma Score using Abbreviated Injury Scale criteria (KTS-AIS), and Kampala Trauma Score using physician opinion (KTS-physician opinion) KTS - Kampala Trauma Score; AIS- Abbreviated Injury Scale; ${ }^{a}$ AIS scores $\geq 3$ were used to calculate serious injuries; ROC - receiver operating characteristics; ${ }^{b}$ Number of serious injuries determined by the emergency department physician providing care were used to calculate serious injuries; SATS - South African Triage Scale 


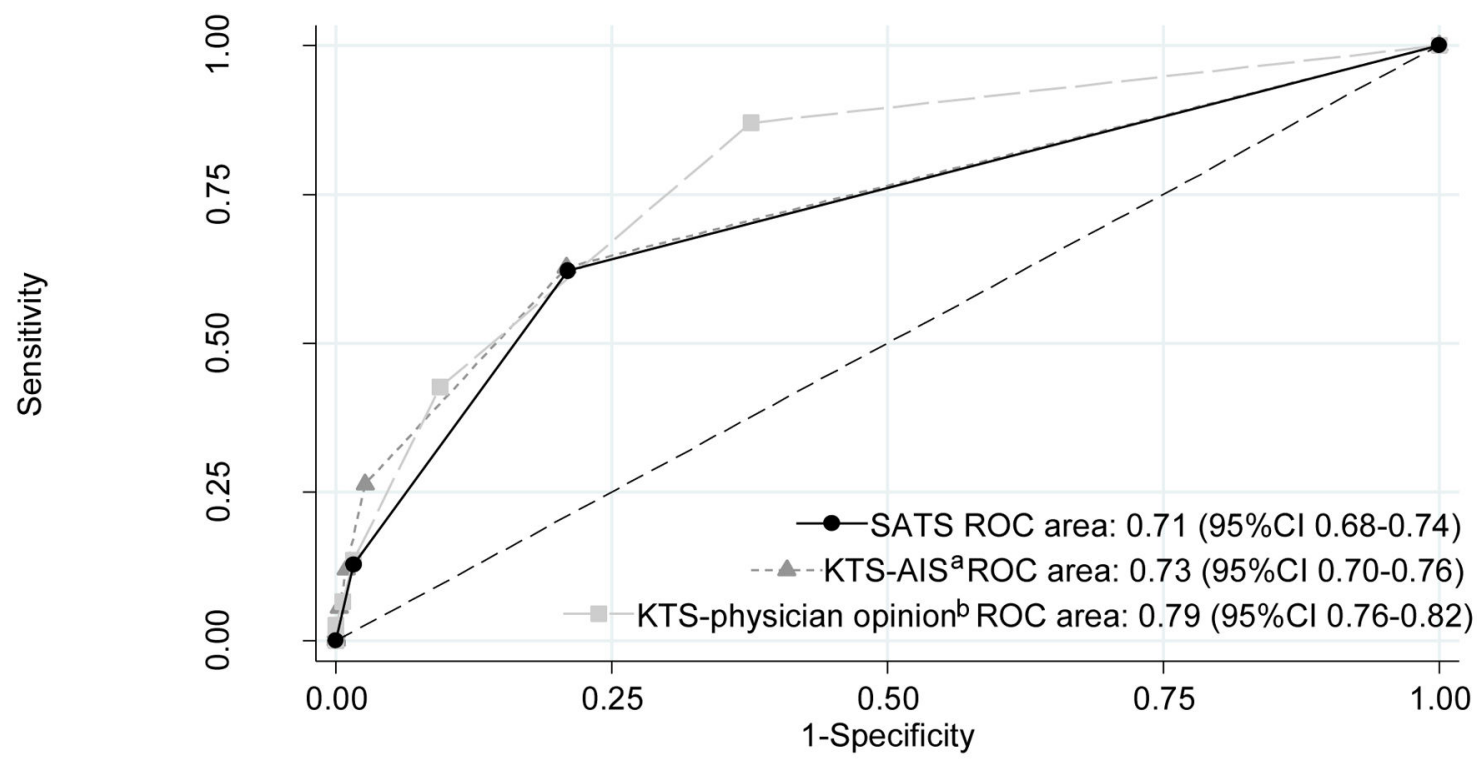

Figure 2. Need for Admission (i.e. hospital ward or theatre) receiver operating characteristics curves for South African Triage Scale (SATS), Kampala Trauma Score using Abbreviated Injury Scale criteria (KTS-AIS), and Kampala Trauma Score using physician opinion (KTS-physician opinion)

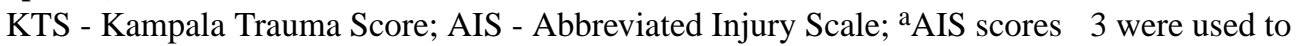
calculate serious injuries; ROC - receiver operating characteristics; ${ }^{\mathrm{b}}$ Number of serious injuries determined by the emergency department physician providing care were used to calculate serious injuries; SATS - South African Triage Scale 
Table 1

\section{Characteristics of study and excluded patients from Komfo Anokye Teaching Hospital} Accident and Emergency Department in Ghana

${ }^{a}$ Excluded patients have 34 missing values for age, 1 missing value for sex, 11 missing values for South African Triage Scale; IQR- interquartile range; ${ }^{b} 387$ patients triaged SATS Green among excluded patients; ${ }^{c}$ Cause of injury collected from January to April 2015 (849 values); RTI- road traffic accident

\begin{tabular}{|c|c|c|c|c|}
\hline \multirow[b]{2}{*}{ Variables $^{a}$} & \multicolumn{2}{|c|}{$\underline{\text { Study Population;n=1053 }}$} & \multicolumn{2}{|c|}{ Excluded Trauma Patients;n=964 } \\
\hline & $\mathbf{n}$ & $(\%)$ & $\mathbf{n}$ & $(\%)$ \\
\hline Time of Admission; median (IQR) & $3: 45 \mathrm{pm}$ & $(11: 00 \mathrm{am}-7: 45 \mathrm{pm})$ & 2:00pm & $(10: 00 \mathrm{am}-6: 30 \mathrm{pm})$ \\
\hline Age; median years (IQR) & 33 & $(26-42)$ & 35 & $(27-49)$ \\
\hline \multicolumn{5}{|l|}{ Sex } \\
\hline Male & 736 & $(70 \%)$ & 673 & $(70 \%)$ \\
\hline Female & 317 & $(30 \%)$ & 290 & $(30 \%)$ \\
\hline \multicolumn{5}{|l|}{ South African Triage Scale ${ }^{b}$} \\
\hline Yellow & 659 & $(62 \%)$ & 610 & $(64 \%)$ \\
\hline Orange & 324 & $(31 \%)$ & 252 & $(26 \%)$ \\
\hline Red & 70 & $(7 \%)$ & 91 & $(10 \%)$ \\
\hline \multicolumn{5}{|l|}{ Cause of Injury ${ }^{c}$} \\
\hline RTI & 549 & $(52 \%)$ & 233 & $(39 \%)$ \\
\hline Fall & 172 & $(17 \%)$ & 131 & $(22 \%)$ \\
\hline Burns & 14 & $(1 \%)$ & 17 & $(3 \%)$ \\
\hline Other & 318 & $(30 \%)$ & 214 & $(36 \%)$ \\
\hline \multicolumn{5}{|c|}{$\begin{array}{l}\text { excluded patients have } 26 \text { missing values for age, } 1 \text { missing value for sex, } 11 \text { missing values for South African Triage Scale; IQR-interquarti } \\
\text { range; }\end{array}$} \\
\hline
\end{tabular}


Table 2

Distribution of patient disposition by Kampala Trauma Score using Abbreviated Injury Scale criteria (KTS-AIS), Kampala Trauma Score using physician opinion (KTSphysician opinion), and South African Triage Scale (SATS)

A\&E - Accident and Emergency Department; KTS-AIS- Kampala Trauma Score calculated using Abbreviated Injury Scale scores $\geq 3 ; *$ KTS score $\leq 4$ denotes a serious injury; KTS-physician opinion- Kampala Trauma Score calculated using the number of serious injuries determined by the emergency department physician providing care; SATS - South African Triage Scale

\begin{tabular}{|c|c|c|c|}
\hline \multirow{2}{*}{\multicolumn{2}{|c|}{ KTS-AIS }} & \multirow{2}{*}{ Admitted to hospital } & \multirow[t]{2}{*}{ Discharged from $A \& E$} \\
\hline & & & \\
\hline 16 & 0 & 146 & 511 \\
\hline 15 & 4 & 143 & 118 \\
\hline 14 & 0 & 56 & 11 \\
\hline 13 & 2 & 25 & 4 \\
\hline 12 & 4 & 16 & 2 \\
\hline 11 & 2 & 3 & 0 \\
\hline 10 & 2 & 1 & 0 \\
\hline 9 & 0 & 1 & 0 \\
\hline 8 & 0 & 0 & 0 \\
\hline 7 & 0 & 0 & 0 \\
\hline 6 & 1 & 0 & 0 \\
\hline 5 & 0 & 1 & 0 \\
\hline
\end{tabular}

\begin{tabular}{llll}
\hline \multicolumn{2}{l}{ KTS-physician opinion } & & \\
\hline 16 & 1 & 51 & 403 \\
15 & 1 & 174 & 182 \\
14 & 2 & 114 & 51 \\
13 & 2 & 27 & 6 \\
12 & 4 & 16 & 4 \\
11 & 1 & 7 & 0 \\
10 & 3 & 1 & 0 \\
9 & 0 & 1 & 0 \\
8 & 0 & 0 & 0 \\
7 & 0 & 0 & 0 \\
6 & 1 & 0 & 0 \\
5 & 0 & 1 & 0 \\
\hline SATS color & & \\
\hline Yellow & 1 & 148 & 510 \\
Orange & 4 & 194 & 126 \\
Red & 10 & 50 & 10 \\
\hline
\end{tabular}

A\&E - Accident and Emergency Department; KTS-AIS- Kampala Trauma Score calculated using Abbreviated Injury Scale scores 23 ; KTSphysician opinion- Kampala Trauma Score calculated using the number of serious injuries determined by the emergency department physician providing care; SATS - South African Triage Scale 


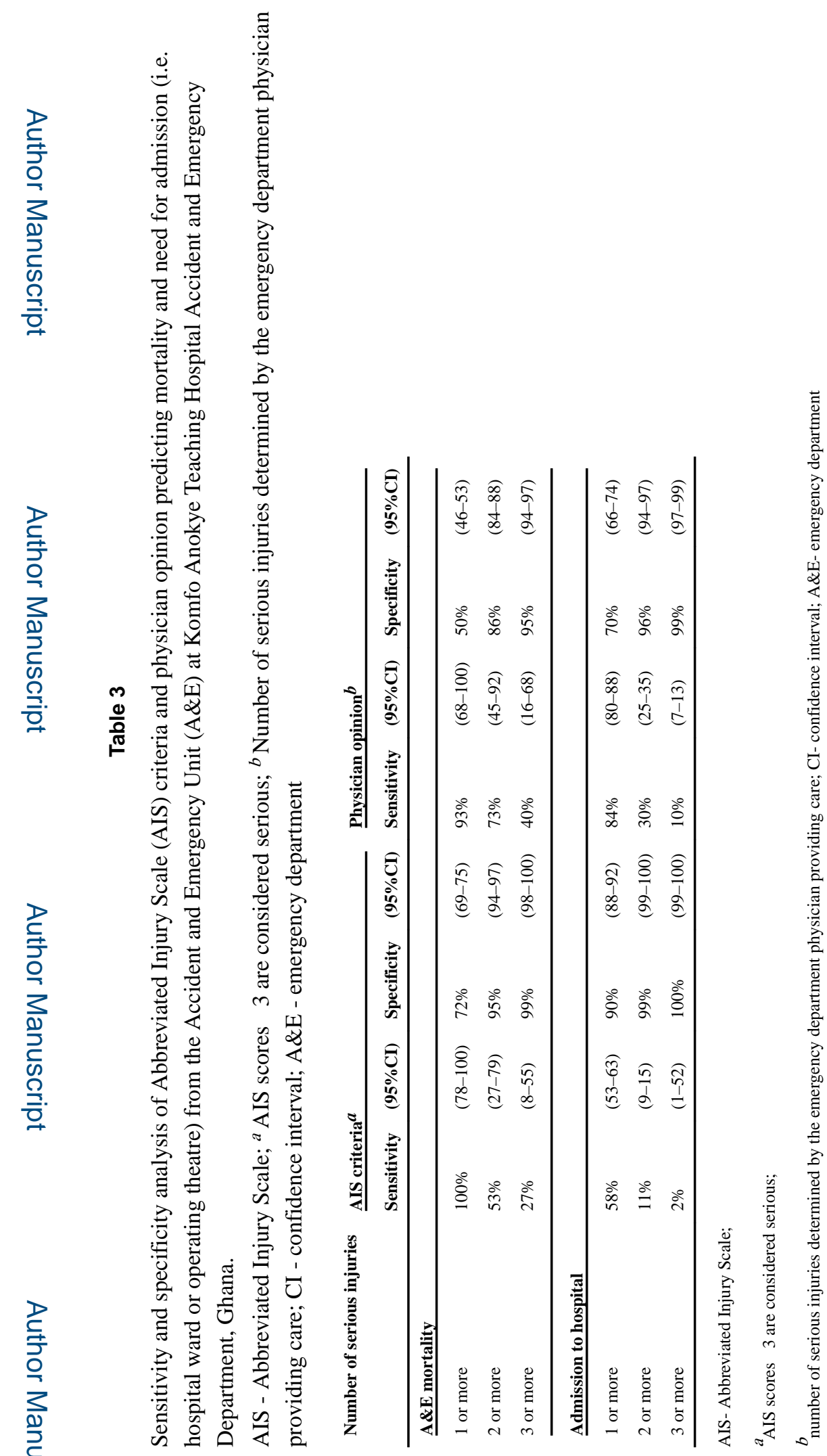

Injury. Author manuscript; available in PMC 2018 January 01. 


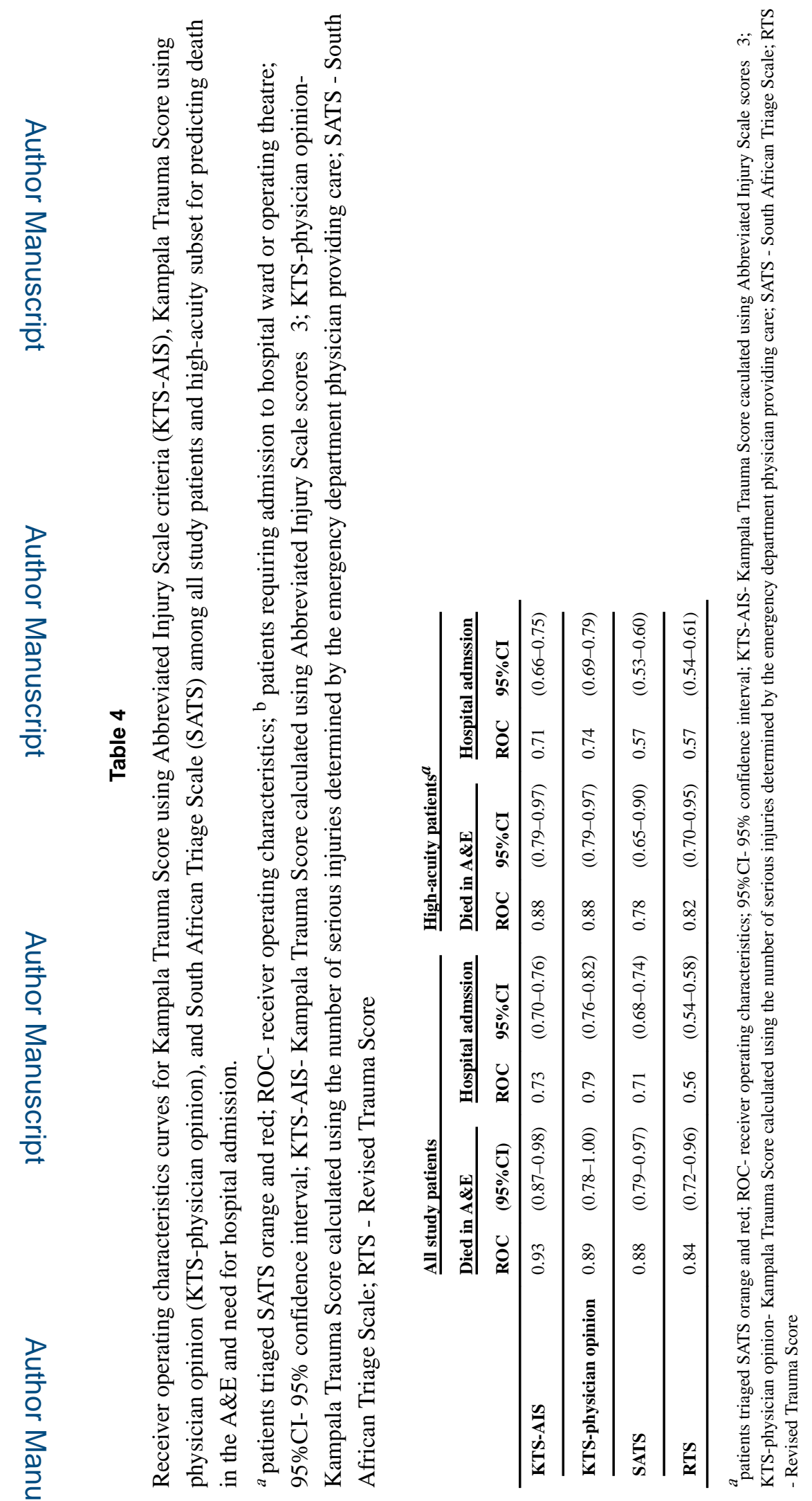

Injury. Author manuscript; available in PMC 2018 January 01. 